\title{
Study of the Non-Linear Stress-Strain Behavior in Ti-Nb-Zr Alloys
}

\author{
Sergio Schneider, Sandra Giacomin Schneider ${ }^{\mathrm{a}}$, Helena Marques da Silva ${ }^{\mathrm{b} *}$, Carlos de Moura Neto $^{\mathrm{b}}$ \\ ${ }^{\text {a}}$ Faculdade de Engenharia Química de Lorena \\ ${ }^{\mathrm{b}}$ ITA, Instituto Tecnológico de Aeronáutica
}

Received: July 19, 2004; Revised: June 27, 2005

\begin{abstract}
The aim of this work is to study the elastic behavior of some Ti-Nb-Zr alloys (Ti-8Nb-13Zr, Ti-13Nb-13Zr, Ti$18 \mathrm{Nb}-13 \mathrm{Zr}$ and $\mathrm{Ti}-41.1 \mathrm{Nb}-7.1 \mathrm{Zr}$ ) developed to biomedical applications. These alloys were produced by arc melting under argon atmosphere. Uniaxial tensile tests, carried out in a MST servo-hydraulic machine, were employed in their mechanical characterization. The occurrence of non-linear stress-strain behavior in the conventional elastic region (total strain values up to $0.2 \%$ ) lead to the Ramberg-Osgood relationship, modified by Hill, in order to analyze that portion of the obtained curves. The present study involves the following properties: initial elastic modulus, tangent modulus, secant modulus, proof stress and the Ramberg-Osgood parameter (n). The results demonstrate that these alloys are not similar with respect to the mechanical behavior. Furthermore, it is shown that the degree of non-linearity in the stress-strain behavior is quantified by the parameter (n).
\end{abstract}

\section{Keywords: mechanical properties, stress-strain behavior, $\mathrm{Ti}-\mathrm{Nb}-\mathrm{Zr}$ alloys}

\section{Introduction}

Titanium alloys are expected to be used as biomaterial due to their superior biocompatibility, corrosion resistance and specific strength compared with the more conventional stainless steels and cobalt-based alloys. In the last years, the principal biomedical titanium alloy has been the Ti-6Al-4V; however on account to toxicity effects caused by $\mathrm{Al}$ and $\mathrm{V}$, new alloys that do not contain these elements are receiving a great deal of attention ${ }^{1,2}$.

Recent biomaterials research has been focused on $\beta$ titanium alloys because processing variables can be controlled to produce selected results. Enhanced properties such as lower modulus of elasticity, increased corrosion resistance, better ductility, and improved notch sensitivity resistance are possible when compared to $\alpha+\beta$ titanium alloys ${ }^{3}$.

Alloys containing elements such as $\mathrm{Nb}, \mathrm{Zr}, \mathrm{Ta}, \mathrm{Pt}$ and $\mathrm{Ti}$ are being extensively evaluated since these are the only five elements that have been identified as producing no adverse tissue reaction ${ }^{4}$.

Evaluating the mechanical and chemical properties is an essential item to design a biomaterial. The mechanical properties are dependent on the microstructure, therefore it is fundamental to control them by modifying microstructure. Moreover, the characterization of biomaterial surfaces is also important due to the osteointregration, which is one important item on biomedical applications ${ }^{5}$.

In the present work Ti-8Nb-13Zr; Ti-13Nb-13Zr; Ti-18Nb-13Zr and Ti-41.1Nb-7.1-Zr alloys are analyzed based on the effect of alloying elements on the mechanical properties. The mechanical properties obtained are initial elastic modulus, proof stress and ultimate tensile strength. Moreover, stress-strain relationship is described based on the modified Ramberg-Osgood equation. It is detected a non-linear stress-strain behavior, even so on the elastic region.

Ramberg-Osgood equation provides a good approximation of the non-linear behavior of some materials, such as stainless steels and aluminum alloys ${ }^{6-8}$. This behavior leads to a relatively difficult design because the stress-strain curves cannot be represented by a linear function. In fact, the specification of an effective modulus is an important aspect that must be incorporated in a further analytical procedure of structural design. The two most commonly definitions about the effective modulus are the secant and tangent moduli ${ }^{6,7}$.
In this work the elastic behavior of some Ti-Nb-Zr alloys (Ti$8 \mathrm{Nb}-13 \mathrm{Zr}$, Ti-13Nb-13Zr, Ti-18Nb-13Zr and Ti-41.1Nb-7.1Zr) are evaluated by the modified Ramberg-Osgood equation. The following properties are obtained: initial elastic modulus, tangent modulus, secant modulus, proof stress and the Ramberg-Osgood parameter (n).

\section{Formulation}

Figure 1 shows the representation of a non-linear stress-strain material behavior and the graphic definition of the parameters used ${ }^{6,8}$.

The value of the initial elastic modulus $\left(\mathrm{E}_{0}\right)$ is obtained by a linear regression least-squares estimate of the slope in the proportional elastic region.

Equation 1 is the usual representation of the non-linear behavior?:

$$
\varepsilon=\frac{\sigma}{E_{0}}+0.002\left(\frac{\sigma}{\sigma_{0.2}}\right)^{n}
$$

where $\varepsilon$ is the strain, $\sigma$ is the stress, $\sigma_{0.2}$ is the $0.2 \%$ proof stress, $\mathrm{E}_{0}$ is the initial elastic modulus and $\mathrm{n}$ is the Ramberg-Osgood parameter which is a measure of the non-linearity of the curve.

The first term of Equation 1 represents the linear behavior and the second represents the non-linear behavior. The component value of non-linear strain in $\sigma_{0.01}$ is $0.0001(0.01 \%)$ and decreases with the reduction of the stress. For low stress values, the non-linear component is not significant when compared to the linear component.

The modified Ramberg-Osgood equation, a three-parameter equation, is used to modeling stress-strain relationship for Ti-Nb-Zr alloys up to $0.2 \%$ total strain. The parameter (n) is obtained using the $0.01 \%$ and $0.2 \%$ proof stresses, which leads to the following equation ${ }^{8}$ :

$$
n=\frac{\ln (20)}{\ln \left(\sigma_{0.2} / \sigma_{0.01}\right)}
$$

where $\sigma_{0.01}$ is the $0.01 \%$ proof stress. It is important to explain that the $n$ value is obtained on the basis of the $0.01 \%$ and $0.2 \%$ proof stresses. The fitting can be seriously inaccurate above this range, leading to a high stress ${ }^{8}$.

The tangent modulus $\left(\mathrm{E}_{\mathrm{t}}\right)$, Equation 3, is defined as the slope of the stress-strain curve at each value of stress. It is defined by Equation 1 as the inverse of the first derivative in respect to strain ${ }^{6}$. 


$$
E_{t}=\frac{E_{0} \sigma_{0.2}}{\sigma_{0.2}+0.002 n E_{0}\left(\frac{\sigma}{\sigma_{0.2}}\right)^{n-1}}
$$

The secant modulus $\left(\mathrm{E}_{\mathrm{s}}\right)$ is defined as the stress to strain ratio at each value of stress which can be also obtained by Equation 1 and represented by the equation ${ }^{6}$ :

$$
E_{s}=\frac{E_{0}}{1+0.002 E_{0} \frac{\sigma^{n-1}}{\sigma_{0.2}^{n}}}
$$

Both definitions, tangent and secant moduli, depend on specifying an appropriate stress-strain level for the particular structure and type of response considered.

\section{Materials and Experimental Procedure}

\subsection{Materials}

Ti-8Nb-13Zr; Ti-13Nb-13Zr; Ti-18Nb-13Zr and Ti-41.1Nb-7.1-Zr alloys were developed in this work to biomedical applications. These alloys were produced from commercially pure materials ( $\mathrm{Ti}, \mathrm{Nb}$ and $\mathrm{Zr}$ ) by arc melting under argon atmosphere in a water-cooled copper hearth. The obtained ingots, with initial diameter of $18 \mathrm{~mm}$, were submitted to sequences of heat treatments at $1000{ }^{\circ} \mathrm{C} / 1 \mathrm{~h}$ (except for Ti-41.1Nb-7.1-Zr, which was treated at $1000{ }^{\circ} \mathrm{C} / 2 \mathrm{~h}$ ) and water quenching, cold-working by swaging in order to achieve a final diameter of $6 \mathrm{~mm}$. Then the alloys were submitted to another heat treatment, which conditions vary for each one, as shown in Table $1^{10-12}$.

\subsection{Experimental procedure}

The mechanical properties of the alloys were evaluated through uniaxial tensile tests; carried out using a servo-hydraulic testing machine, model MTS $810.23 \mathrm{M}$. Mechanical tests were performed according to standard test method ASTM E8 to determine the ultimate tensile strength, $0.2 \%$ proof stress and elongation, and ASTM E111 to determine the initial elastic modulus. Furthermore, the stress-strain relationship is studied based on the Ramberg-Osgood equation, in the elastic region, which parameters were used to determine the tangent and secant modulus.

\section{Results and Discussions}

The experimental stress-strain curves for the materials tested are plotted in Figure 2. Mechanical properties values are summarized in Table 2.

In general, the obtained values of initial elastic moduli and ultimate tensile strength, for the studied alloys, are in a range adequate for biomedical applications, according to Niinomi (1998). It is important to emphasize that the Ti-13Nb-13Zr alloy presents lower initial elastic modulus and higher ultimate tensile strength, when compared with the other studied alloys.

Although the alloying elements and thermomechanical processing change the final mechanical properties, there is no direct relation of

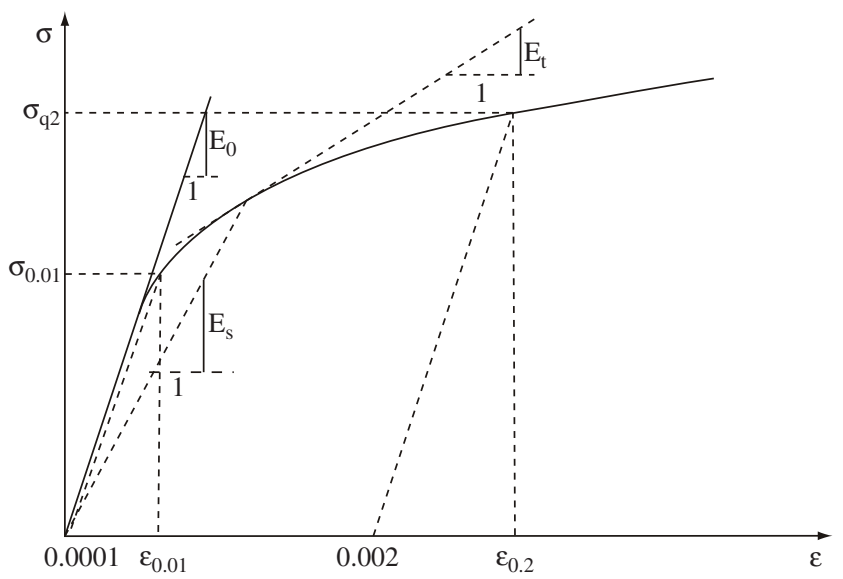

Figure 1. Typical non-linear stress-strain curve.

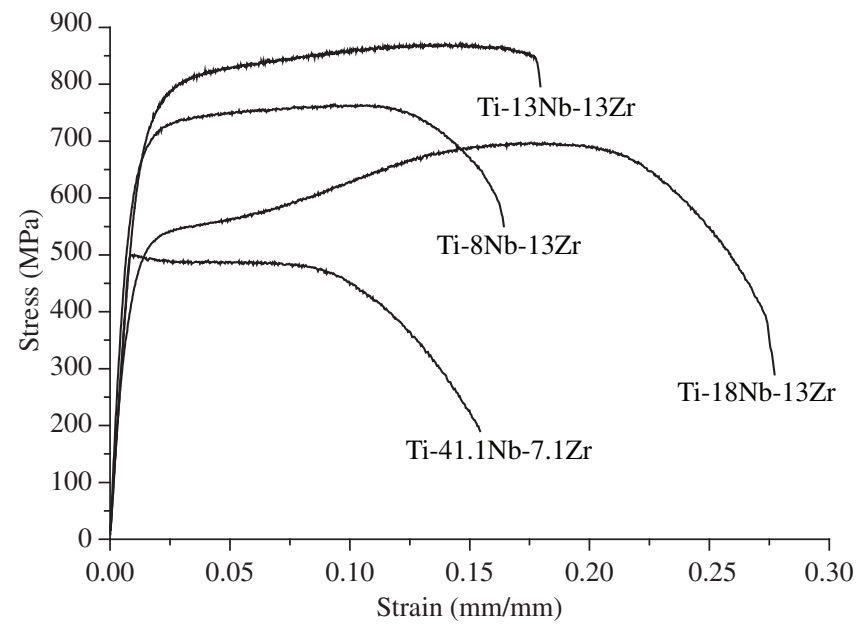

Figure 2. Full stress-strain curves for Ti-Nb-Zr alloys.

Table 1. Conditions of heat treatment after cold - working.

\begin{tabular}{lcc}
\hline Alloys & \multicolumn{2}{c}{ Conditions } \\
& Temperature $\left({ }^{\circ} \mathrm{C}\right)$ & Time $(\mathrm{h})$ \\
\hline Ti-8Nb-13Zr & 900 & 0.5 \\
Ti-13Nb-13Zr & 1000 & 1.0 \\
Ti-18Nb-13Zr & 900 & 0.5 \\
Ti-41.1Nb-7.1-Zr & 1000 & 2.0 \\
\hline
\end{tabular}

\begin{tabular}{|c|c|c|c|c|c|c|c|}
\hline Alloy & $\mathrm{E}_{0}(\mathrm{GPa})$ & $\sigma_{0.01}(\mathrm{MPa})$ & $\sigma_{0.2}(\mathrm{MPa})$ & $\sigma_{\mathrm{u}}(\mathrm{MPa})$ & $\varepsilon_{0.2}$ & $\mathrm{n}$ & Elongation (\%) \\
\hline Ti-8Nb-13Zr & 88.8 & 285 & 551 & 763 & 0.00805 & 4.56 & 15 \\
\hline Ti-13Nb-13Zr & 61.9 & 440 & 646 & 870 & 0.01234 & 7.80 & 16 \\
\hline Ti-18Nb-13Zr & 70.4 & 191 & 374 & 698 & 0.00733 & 4.46 & 26 \\
\hline Ti-41.1Nb-7.1Zr & 64.7 & 474 & 499 & 499 & 0.00969 & 58.28 & 15 \\
\hline
\end{tabular}

Table 2. Mechanical properties of the Ti-Nb-Zr alloys. 
the effect of the percentage of $\mathrm{Nb}$ on the elastic moduli. For instance, comparing the alloys of the system Ti-XNb-13Zr, the one that has the lowest percentage of $\mathrm{Nb}(\mathrm{Ti}-8 \mathrm{Nb}-13 \mathrm{Zr})$ presents the highest elastic modulus (88.0 GPa), whereas the lowest elastic modulus (61.9 GPa) was obtained for the intermediate composition alloy (Ti-13Nb-13Zr). It is noted that the elongation depends on the amount of $\mathrm{Nb}$, e.g., higher percentage of $\mathrm{Nb}$ leads to higher elongation, but this effect is not observed on the other properties, as it can see on Table 1.

Ti-41.1Nb-7.1Zr alloy presents an initial elastic modulus near to the Ti-13Nb-13Zr. However, it has low mechanical resistance when compared with the others. This alloy does not have the capacity of work hardening, and its characteristics can be considered typically as elastic, perfectly plastic.

Figure 3 is plotted in order to obtain a better interpretation of the stress-strain relationship on the elastic region for the studied alloys. It is observed that each alloy presents different characteristics, such as initial elastic modulus given by the slope of the curve, up to proportional elastic limit, in which the non-linear stress-strain behavior is verified.

The stress-strain relationships of these alloys are fitted based on the Ramberg-Osgood equation. The parameters are summarized on Table 1. The degree of non-linearity is quantified via parameter (n), e.g., low value of $n$ corresponds to the facility of occurring a non-linear behavior. Observing Figure 3 it is possible to identify the non-linear behavior, which is more accentuated in Ti-8Nb-13Zr and Ti-18Nb-13Zr alloys.

Considering this effect, this work proposes to analyze the stiffness aspect of the materials based on the secant and tangent moduli, which depend on the stress applied according to described in Equations 3 and 4 . Figures 4 and 5 show the influence of the stress on the tangent and secant moduli, respectively, on the elastic region.

In the proportional region the secant and tangent moduli are equal to the initial elastic modulus $\left(\mathrm{E}_{0}\right)$. This fact indicates that the stiffness aspect of the materials is constant. The alloy containing $41.1 \% \mathrm{Nb}$ presents the higher linearity $(n=58.28)$ without any change on the modulus until around $470 \mathrm{MPa}$. The behavior of stiffness possesses one characteristic for each one of the alloys when they are submitted to higher values of stress than proportional elastic limit.

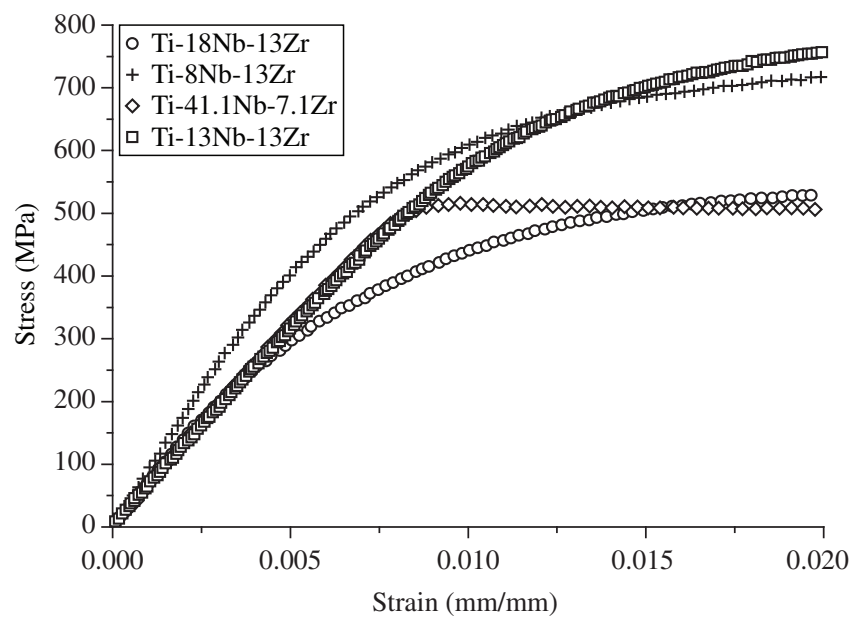

Figure 3. Stress-strain curves for Ti-Nb-Zr alloys cut-off around $2 \%$ total strains.
The alloys containing 8 and $18 \%$ of $\mathrm{Nb}$ (n values equal to 4.56 and 4.46 respectively) possesses a similar behavior. Comparing them with the others alloys the decreasing on stiffness is more evident.

The alloy with $18 \%$ of $\mathrm{Nb}$ possesses one interesting characteristic related to the tangent and secant moduli. The initial modulus is higher than the one of the alloy with $13 \%$, but increasing the value of stress this modulus becomes lower.

It is known that one of the main aspects, related to mechanical properties for biomaterials, is the elastic modulus. Then, this study shows that it is possible to obtain additional information more representative when the effective modulus is considered for representation of the mechanical behavior of the materials.

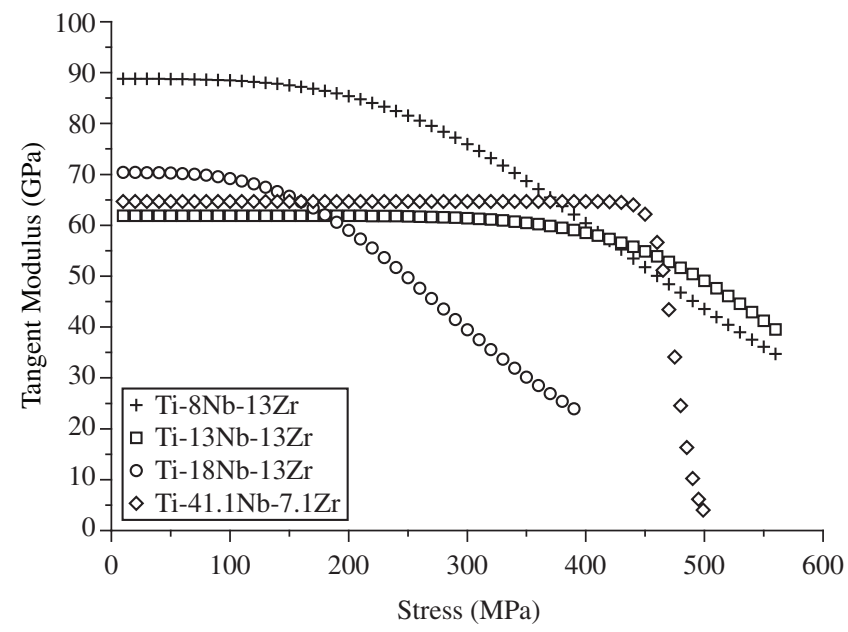

Figure 4. Tangent modulus versus stress for Ti-Nb-Zr alloys.

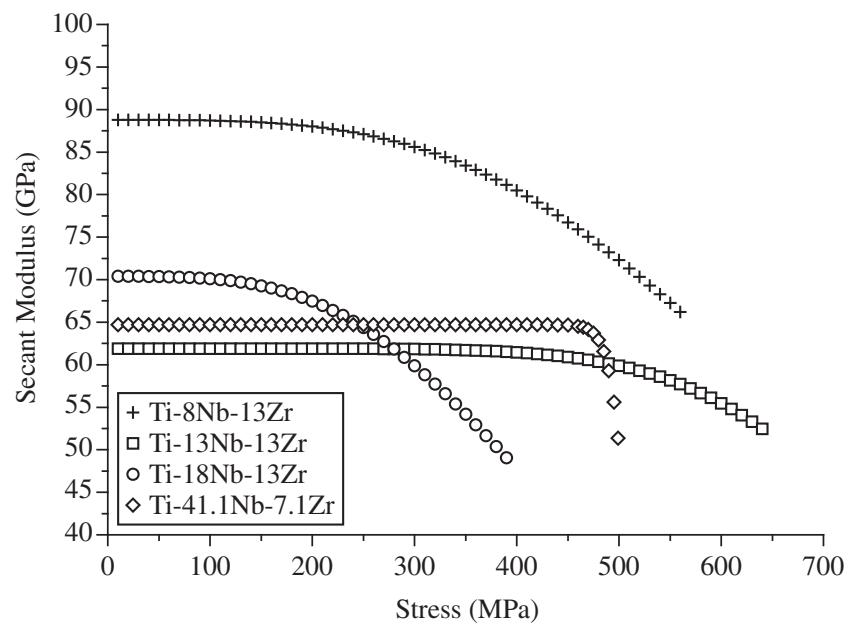

Figure 5. Secant modulus versus stress for Ti-Nb-Zr alloys. 


\section{Conclusions}

The analysis of Ti-8Nb-13Zr; Ti-13Nb-13Zr; Ti-18Nb-13Zr and $\mathrm{Ti}-41.1 \mathrm{Nb}-7.1-\mathrm{Zr}$ alloys developed in this work shows a typical non-linear stress-strain behavior, even so in the elastic region. The modified Ramberg-Osgood equation fits adequately the stress-strain relationship, describing the non-linear effect. The degree of non-linearity is quantified via parameter (n), that varies from 4.46 to 58.28, according to non-linear behavior of the materials.

The values of secant and tangent moduli allow one future determination of an effective modulus, that is important to better evaluate the characteristics of the materials and to define their application as a biomaterial. Moreover, as these parameters are dependent on stress and strain values it is possible to know the behavior of the material for different levels of stress.

Therefore, the methodology employed in this work is available to obtain additional details to characterize new alloys developed for application as biomaterial. It is hoped that the present results and discussions provide helpful information for the fundamental understanding of the real behavior of the materials.

\section{References}

1. Niinomi M. Mechanical properties of biomedical titanium alloys. Materials Science and Engineering A. 1998; 243:231-236.

2. Long HJRM. Review titanium alloys in total joint replacement - a Materials Science Perspective. Biomaterials. 1998; 19:1621-1639.
3. Diseg JA. Titanium alloys for fracture fixation implants. International Journal of the Injured-INJURY., 31, Suppl. 4, S-D14-17, 2000.

4. Geetha M, Singh AK, Muraleedharan K, Gogia AK, Asokamani R. Effect of thermomechanical processing on microstructure of a Ti-13Nb-13Zr alloy. Journal of Alloys and Compounds. 2001; 329:264-271.

5. Ratner BD, Hiffman AS, Schoen FJ, Lemons JE. An introduction to materials in medicine. San Diego: Academic Press; 1996.

6. Berg, Van Den, GJ. The effect of the non-linear stress-strain behavior of stainless steels on member capacity. Journal of Constructional Steel Research. 2000; 54:135-160.

7. Chryssanthopoulos, MK. A method for predicting the flexural response of tubular members with non-linear stress-strain characteristics. Journal of Constructional Steel Research. 2001; 57:1197-1216.

8. Rasmussen KJR. Full-range stress-strain curves for stainless steels alloys. Journal of Constructional Steel Research. 2003; 59:47-61.

9. Hill BN. Determination of stress-strain relationships from offset strength values. NACA technical note ${ }^{\circ} 927 ; 1944$.

10. Schneider SG, Nunes CA, Ferreira NAM, Bressiani JC. Microstructure of arc-melted and heat-treated Ti-13Nb-13Zr alloy. Acta Microscopica, suppl. A; 2001. p. 57-58.

11. Schneider, S. G. Obtenção e caracterização da liga Ti-13Nb-13Zr para aplicação como biomaterial. São Paulo, 2001. Tese de doutorado - Instituto de Pesquisas Energéticas e Nucleares (IPEN).

12. Silva HM, Schneider SG, Moura Neto C. Microstructure of arc-melted and heat-treated Ti-8Nb-13Zr and Ti-18Nb-13 Zr alloys. Acta Microscopica, suppl. C. $2003 ; 12: 7$. 